\title{
Modelling of spectral properties and population kinetics studies of inertial fusion and laboratory-astrophysical plasmas
}

\author{
E Mínguez R Florido R Rodríguez J M Gil J G Rubiano \\ M A Mendoza G Espinosa and P Martel
}

\begin{abstract}
Fundamental research and modelling in plasma atomic physics continue to be essential for providing basic understanding of many different topics relevant to high-energy-density plasmas. The Atomic Physics Group at the Institute of Nuclear Fusion has accumulated experience over the years in developing a collection of computational models and tools for determining the atomic energy structure, ionization balance and radiative properties of, mainly, inertial fusion and laser-produced plasmas in a variety of conditions. In this work, we discuss some of the latest advances and results of our research, with emphasis on inertial fusion and laboratory-astrophysical applications.
\end{abstract}

(Some figures may appear in colour only in the online journal)

\section{Introduction}

Fundamental research and modelling in plasma atomic physics continue to be essential for providing both basic understanding and advancing on many different topics relevant to high-energy-density systems community [1]. In the most general scenario, the plasmas are not in local thermodynamic equilibrium (LTE), which means many atomic propertiessuch as cross-section of all relevant collisional and radiative atomic processes and atomic energy levels-need to be known to properly describe and diagnose the plasma conditions. The basis for most non-LTE (NLTE) simulations is the collisionalradiative (CR) model [2], which describes each ion charge state in the plasma in terms of a number of atomic levels. The distribution of atomic populations among the levels is determined by solving a set of coupled rate equations whose generation requires calculating all transition rates among the atomic levels. Once the population distributions have been obtained, material and radiative properties-such as opacities, emissivities, specific energies and other quantities of interest for the equation of state-can be calculated.
In this connection, the Atomic Physics Group at the Institute of Nuclear Fusion (DENIM) in Spain has accumulated experience over the years in developing a collection of computational models and tools for determining the atomic energy structure, ionization balance and radiative properties of, mainly, inertial confinement fusion (ICF) and laserproduced plasmas in a variety of conditions. In this work, we will focus on some of the latest advances and results of our research, with emphasis on ICF and, also, laboratory-astrophysical applications. In the following section we briefly describe the most recently developed models and codes, i.e. ABAKO/RAPCAL [3,4] and ATMED [5]. ABAKO/RAPCAL was designed to perform detailed atomic kinetics and radiative properties calculations over a wide range of temperature and density, including the coronal, LTE and non-LTE regimes. Meanwhile, the new averageatom screened hydrogenic model, ATMED, was aimed for providing fast estimates and potential in-line hydrodynamical calculations of emissivities and opacities for plasmas under LTE conditions. In sections 3 and 4, we report on recent applications of ABAKO/RAPCAL to two completely different 
kind of laboratory-plasma experiments. In the first one, connected to laboratory astrophysics, radiative properties calculated from RAPCAL are used to characterize the radiative blast waves launched in xenon clusters. In the second one, relevant for the ICF community, we discuss the use of ABAKO as part of physics model employed for the spectroscopic analysis of shock-ignition implosions. Finally, conclusions are presented.

\section{ABAKO/RAPCAL and ATMED computational packages}

ABAKO is a CR model that can be applied to low-to-high $Z$ ions for a wide range of laboratory-plasma conditions: coronal, LTE or NLTE, and optically thin or thick plasmas. It combines a set of analytical approximations to atomic rates, which yield substantial savings in computer running time, still comparing well with more elaborate codes and experimental data. Autoionizing states are explicitly included in the kinetics in a fast and efficient way. Radiation transport effects due to line trapping in the plasma are taken into account via geometrydependent escape factors. Also, since the atomic kinetics problem often involves very large sparse matrices, an iterative method is used to perform the matrix inversion. It has been shown that ABAKO results compare well with customized models and simulations of ion population distributions. A detailed description of the model can be found in [3].

Meanwhile, the RAPCAL [4] code determines several relevant plasma radiative properties such as the frequencydependent opacities and emissivities, mean and multigroup opacities, source functions, radiative power losses, specific intensities and plasma transmission. For line transitions, RAPCAL uses a Voigt profile including natural and Doppler broadenings, unresolved-transition-array (UTA) corrections, as well as an approximate formula to account for the electronimpact width.

In the following two sections, we discuss the use of ABAKO/RAPCAL for the study and investigation of two completely different kinds of laboratory-plasma experiments, one connected to laboratory astrophysics and the other one to ICF.

Furthermore, the ATMED code was aimed for providing fast calculations of the photon energy dependent opacity as well as the Rosseland and Planck mean opacities for single element and mixture hot dense plasmas under LTE. Typical ATMED running times are of the order of seconds. The code was developed within the framework of an average atom model. The needed atomic data are computed using a relativistic screened hydrogenic model based on a new set of universal screening constants including $\mathrm{j}$-splitting [5]. These screening constants were obtained from the fit to a wide database of high-quality atomic energies, ionization potentials and transition energies taken from [6,7]. The opacity calculations take into account the bound-bound, bound-free, free-free contributions, as well as scattering processes. The line absorption cross-sections are computed using a new analytical expression for oscillator strengths based on relativistic screened-hydrogenic wave functions. Line shapes include natural, Doppler and electron collisional

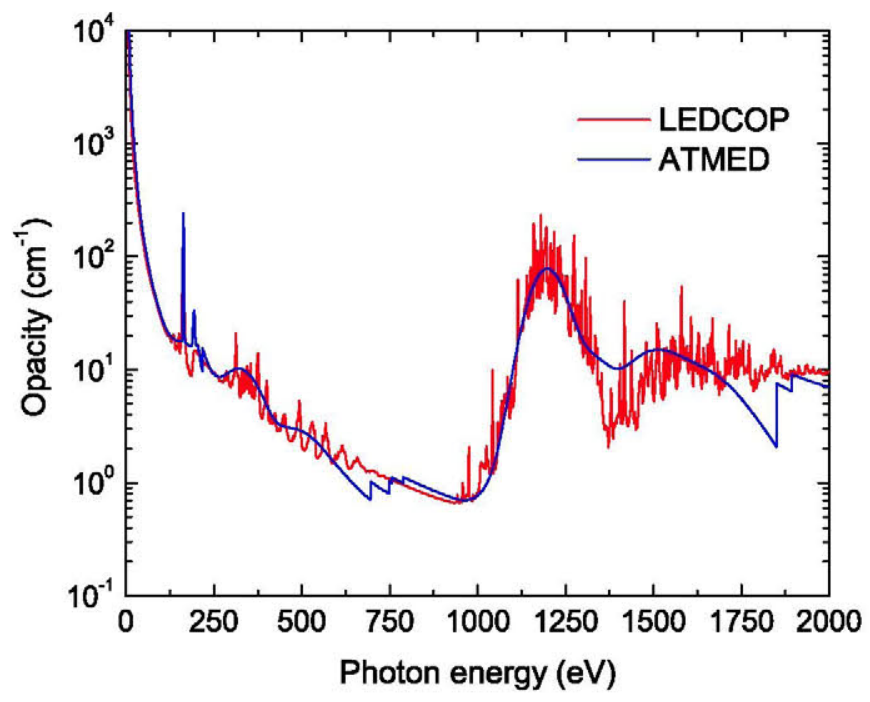

Figure 1. Frequency-dependent opacity for a $\mathrm{Be}(99.1 \%)-\mathrm{Cu}(0.9 \%)$ plasma mixture at $250 \mathrm{eV}$ and $0.1 \mathrm{~g} \mathrm{~cm}^{-3}$. A comparison between ATMED and LEDCOP [9] is shown.

broadenings. To obtain a more realistic value of the Rosseland mean opacity, an additional broadening of the bound-bound transitions has been included by considering the fluctuations of the occupations numbers into the atomic shells. ATMED also provides the plasma equation of state and shock Hugoniot curves. The model has been successfully tested and, in spite of its simplicity, it is able to predict the correct order of magnitude of quantities of interest, so it can be used to model radiative transport phenomena in hydrodynamic codes and experiments in an approximate way. A detailed discussion of many applications of ATMED can be found in [8]. Here, as a case of illustration, we show in figure 1 the frequencydependent opacity corresponding to a $\mathrm{Be}-\mathrm{Cu}$ plasma mixture. A calculation like this could be of interest for simulations of indirect-drive fusion capsules, in which $\mathrm{Cu}$-doped beryllium has been used as ablator material.

\section{An application to laboratory-astrophysical plasmas: characterizing radiative blast waves created in the laboratory}

The main goal of laboratory astrophysics is to design and perform reproducible and well-characterized experiments, and then use hydrodynamical simulations and scaling arguments to both explain and predict actual astrophysical phenomena. Among many others, one particular area of interest within laboratory astrophysics is that of radiative shocks [10,11], which are observed around astronomical objects in a wide variety of forms, e.g. accretion shocks, pulsating stars, supernovae in their radiative cooling phase, etc.

In this work, we report on atomic kinetics and radiative properties calculations in connection to radiative blast waves launched in xenon clusters [12,13]. Ordinarily gases absorb only a small fraction of the laser energy. However, if atomic clustered gases are used, the absorption increases dramatically and a hot $\left(T_{\mathrm{e}} \sim \mathrm{keV}\right)$, high-energy-density plasma is formed and, eventually, a cylindrical blast wave generated [14]. Using 

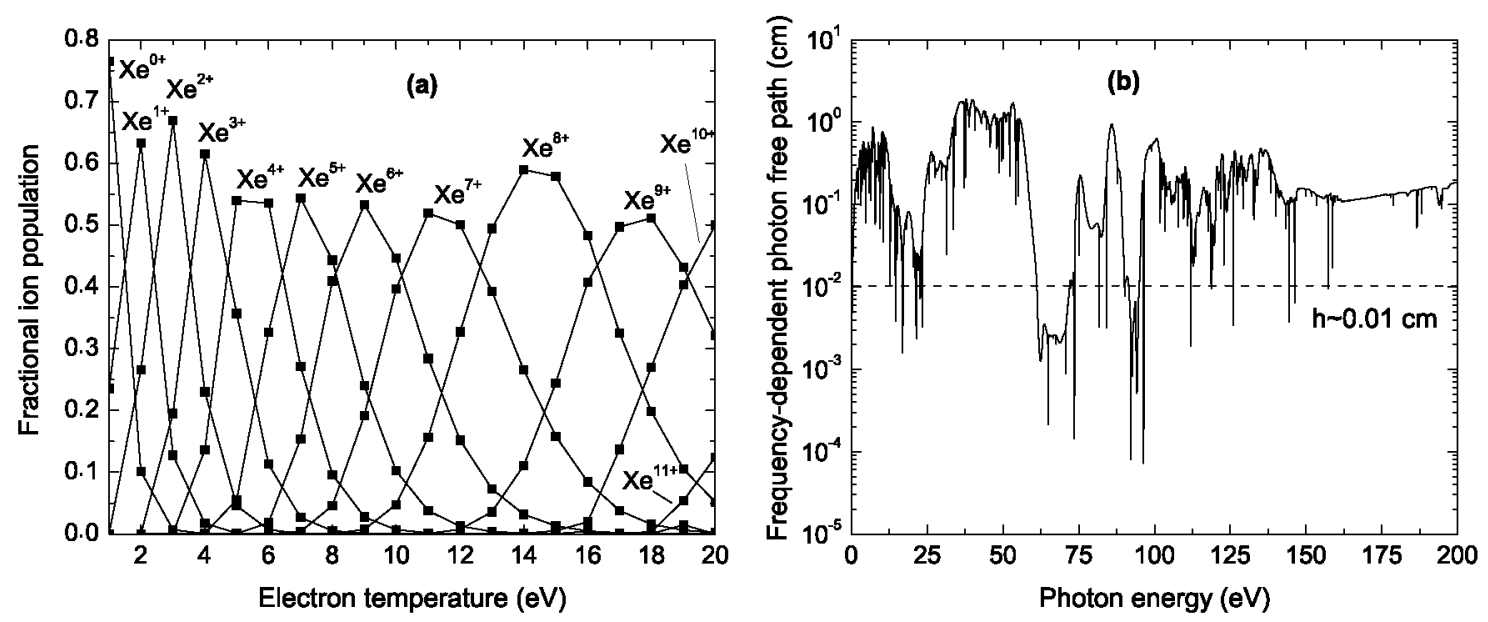

Figure 2. (a) Xe charge state distribution as a function of temperature for a mass density of $5 \times 10^{-4} \mathrm{~g} \mathrm{~cm}^{-3}$. (b) Frequency-dependent photon free path for a xenon plasma at $10 \mathrm{eV}$ and $5 \times 10^{-4} \mathrm{~g} \mathrm{~cm}^{-3}$. A dashed line indicating the characteristic dimension of the system has been added for comparison.

clusters as a target medium, radiative blast waves can be launched even with laser energies as low as $100 \mathrm{~mJ}$, provided that the pulse duration is short enough for an efficient energy coupling into clusters before they explode (which requires subpicosecond lasers).

In the experiments here discussed, xenon clusters were irradiated at average gas densities of 0.16 and $0.25 \mathrm{~kg} \mathrm{~m}^{-3}$ with femtosecond laser pulses of $440 \mathrm{~mJ}$ and $360 \mathrm{~mJ}$, respectively. The evolution and morphology of the blast waves were monitored using imaging interferometric and Schlieren techniques. Time histories of electron density radial profiles were also determined from interferometric measurements. ABAKO/RAPCAL was used for a better understanding and characterization of these radiative blast waves $[15,16]$. Typical mass densities and electron temperatures of these xenon plasmas are expected to be in the range $10^{-5}-10^{-3} \mathrm{~g} \mathrm{~cm}^{-3}$ and $1-20 \mathrm{eV}$, respectively. A first step for the characterization of the xenon plasmas was to perform a population kinetics study and compute basic quantities such as the average ionization and ion population distribution for such plasma conditions. For the given ranges of temperature and density, and within the steady-state assumption, it is difficult to know a priori whether the population distributions of xenon plasmas will be those characteristic of a coronal, LTE or NLTE regime. This fact prevents the use of a simplified coronal model as well as the Saha-Boltzmann (SB) equations, at least until a first atomic kinetics study has been carried out based on collisionalradiative calculations. At this point, it must be noted that such xenon atomic kinetics calculations are challenging, since for the temperatures and densities of interest, xenon will be only a few times ionized and the calculations will involve complex ions with a large number of bound electrons. As the number of bound electrons increases, the number of energy levels and electronic configurations do dramatically and some averaging technique must be used to keep the problem manageable. Here, we took advantage of the proper balance existing in $\mathrm{ABAKO}$ between accuracy and computational cost to obtain reasonable estimates of average ionization and ion population distributions. Additionally, we made a careful selection of electronic configurations to be included following the criteria discussed in [16]. For each CR calculation, the model included about ten xenon ions, which yields a CR matrix with $\sim 70000$ energy levels. As illustration, in figure 2(a) the charge state distribution (CSD) of xenon is plotted as a function of the electron temperature for a fixed mass density of $5 \times 10^{-4} \mathrm{~g} \mathrm{~cm}^{-3}$, characteristic of blast waves in xenon clusters. As seen, population is mainly distributed among four different charge states for a given temperature value within the range of interest.

Also, the conditions to define an optically thin, radiative regime for laboratory astrophysics experiments relevance to radiative supernova remnants were formulated in [17]: (a) for the radiative flux to escape from the blast wave, the mean free path of the photons, $\lambda_{\text {ph }}$, must be larger than a characteristic size scale of the system, $h$; and (b) the radiative cooling time, $\tau_{\text {rad }}$, must be shorter than the convective transport time, $\tau_{\text {conv }}=h / s$, where $s$ is the plasma sound speed. In this regard, measurements indicate that blast waves in clustered xenon reached the needed conditions to enter the radiative flux regime-defined as the situation in which the radiative energy flux is greater than the material energy flux, thus playing an important role in the evolution of the blast wave. Nevertheless, a theoretical confirmation of this fact is desirable and here we show as ABAKO/RAPCAL becomes useful to carry out this study. The frequency-dependent free path of photons was computed for a mass density of $\rho=5 \times 10^{-4} \mathrm{~cm}^{-3}$ and an electron temperature of $T_{\mathrm{e}}=10 \mathrm{eV}$ (see figure $2(b)$ ), which are approximated values for the upstream medium according to measurements and hydrodynamical simulations $[12,13]$. Characteristic size of the xenon blast waves discussed here is $h \approx 0.01 \mathrm{~cm}$, so, as observed in figure $2(b)$, the upstream medium can be assumed as optically thin for almost the entire range of photon energies, although some absorption effects are expected in the interval from 60 to $100 \mathrm{eV}$. We also computed the radiative cooling time $\tau_{\text {rad }}$ [17] for several temperature and density values within the range of interest and for all cases $\tau_{\text {rad }}$ was found to be less than $\tau_{\text {conv }} \approx 1 \mu \mathrm{s}$, which is the typical convective transport time of the xenon blast waves. 

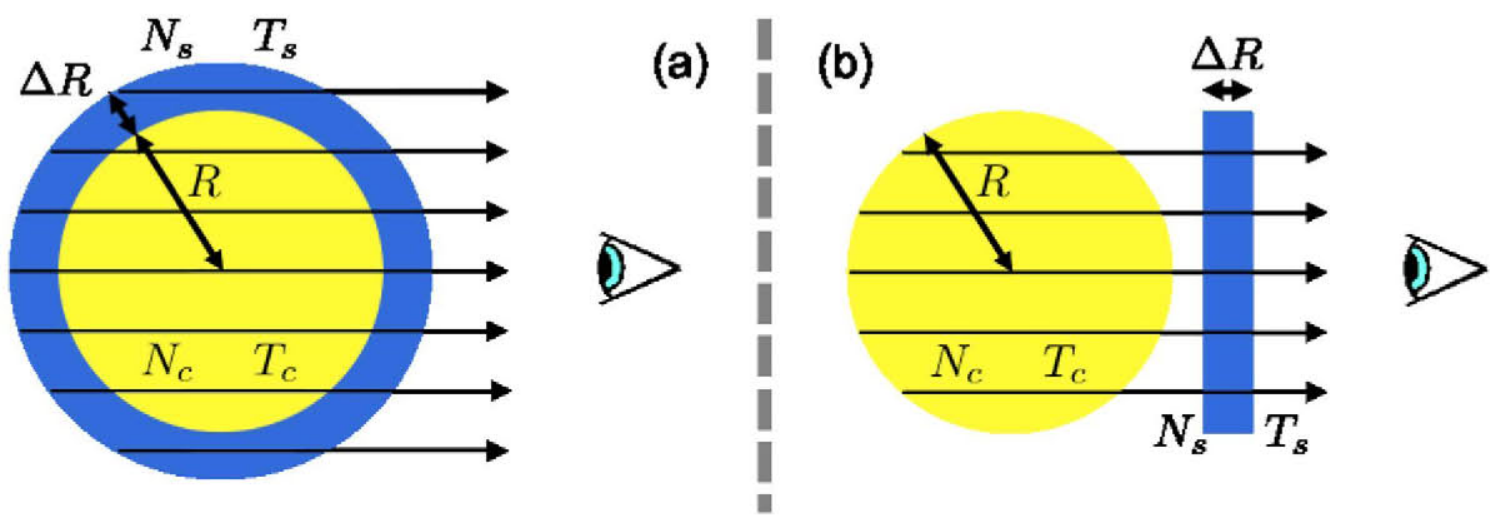

Figure 3. (a) Schematic illustration of the model used to analyse the data. (b) Simpler geometry with analytical solution. Figure adapted from [20]. Copyright (2011) by American Physical Society.

Therefore, with the only exception of a photon energy interval of width $\sim 60 \mathrm{eV}$, the theoretical analysis performed using ABAKO/RAPCAL allows us to classify the xenon blast waves within the optically thin, radiative regime. This conclusion agrees with what had been previously suggested based on experimental data.

The results discussed so far were obtained assuming steady state. Therefore, for them to be meaningful, the validity of this assumption for the case of study must be checked and possible time-dependent effects discarded. A key quantity for this analysis is the time scale of the most frequent atomic process in the plasma, $\tau_{a}[18]$. Time-independent models can be acceptably accurate if the characteristic time $t_{\text {plasma }}$ for the change in the local plasma conditions is significantly larger than $\tau_{a}$, i.e. $t_{\text {plasma }} \gg \tau_{a}$. When this inequality holds true, it can be considered that the populations distributions adjust almost instantaneously to the changing conditions of the plasma. For the case of blast waves experiments in xenon, it can be inferred from data that $t_{\text {plasma }} \sim 1$ ns. Moreover, calculations made with $\mathrm{ABAKO}$ for densities from $10^{-4}$ to $10^{-3} \mathrm{~g} \mathrm{~cm}^{-3}$ and temperatures from 1 to $20 \mathrm{eV}$ give $\tau_{a}$ values which are slightly less than $t_{\text {plasma. }}$. This means that the results shown above must be taken with caution and considered only for the purpose of qualitative analyses. An accurate study would require full time-dependent calculations.

\section{An application to ICF plasmas: spectroscopic diagnosis of shock-ignition implosions}

ABAKO has been used as part of the physics model employed for the first spectroscopic analysis of shock-ignition implosions. The results have been discussed and published in $[19,20]$. Here, we briefly emphasize the main results. Shock ignition [21] is an approach to ICF that relies on the lowadiabat implosion of a thick-wall shell target that creates a high areal-density fuel assembly, which is subsequently driven to ignition by a spherically convergent shock wave. Initial shock-ignition experiments at the OMEGA laser facility were successfully performed [22]. Furthermore, shock-ignition has aroused significant interest in the ICF community and several recent studies have been performed for possible shockignition experiments at the National Ignition Facility [23] in the United States as well as in the future HiPER [24] and Laser Megajoule [25] facilities in Europe.

The shock-ignition implosions discussed here and the associated spectroscopic analysis were performed at the OMEGA laser facility and led by Mancini from the University of Nevada, Reno. They employed plastic shell targets that had an internal radius of $387 \mu \mathrm{m}$, a wall thickness of $40 \mu \mathrm{m}$ and an outer aluminum coating of $0.1 \mu \mathrm{m}$ for sealing purposes. They were filled with $20 \mathrm{~atm}$ of $\mathrm{D}_{2}$ and $0.072 \mathrm{~atm}$ of $\mathrm{Ar}$, which was used for spectroscopic diagnosis. The observed argon radiation emission includes $\mathrm{K}$-shell $\mathrm{x}$-ray line transitions that span the photon energy range from 3000 to $4400 \mathrm{eV}$.

The model used to analyse the data is schematically illustrated in figure $3(a)$. It consists of a uniform sphere to characterize the state of the implosion core surrounded by a uniform concentric shell to account for the compressed shell confining the core. The parameters of the model are the core radius $R$, electron temperature $T_{\mathrm{c}}$, electron density $N_{\mathrm{c}}$, the compressed shell thickness $\Delta R$, electron temperature $T_{\mathrm{s}}$ and electron density $N_{\mathrm{s}}$. On the one hand, the value of $R$ can be computed from $N_{\mathrm{c}}$ assuming mass conservation; this assumption relies on the approximation that all of the core mass contributes to the emission of radiation. On the other hand, the value $\Delta R$ is calculated from the values of $R$ and $N_{\mathrm{s}}$, as well as the fraction $\eta$ of shell mass that is imploded, which is estimated from hydrodynamic simulations of the implosions [19]. The uniform model approximation for the spherical shell requires that the same imploded shell mass that contributes to the attenuation of the core radiation also contributes to the selfemission of the shell. To calculate the emergent intensity distribution for this model, we integrate the radiation transport equation for each photon energy along chords parallel to the direction of observation, and then further integrate all individual chord contributions to obtain the space-integrated spectrum for comparison with the experimental spectrum.

The frequency-dependent emissivity and opacity of the core and shell were calculated with population number densities from ABAKO. All bound-bound, bound-free and free-free contributions from the argon-deuterium plasma in the core and the carbon-hydrogen plasma in the compressed shell within the spectral range of the measurements were included. For a given set of plasma temperature and 

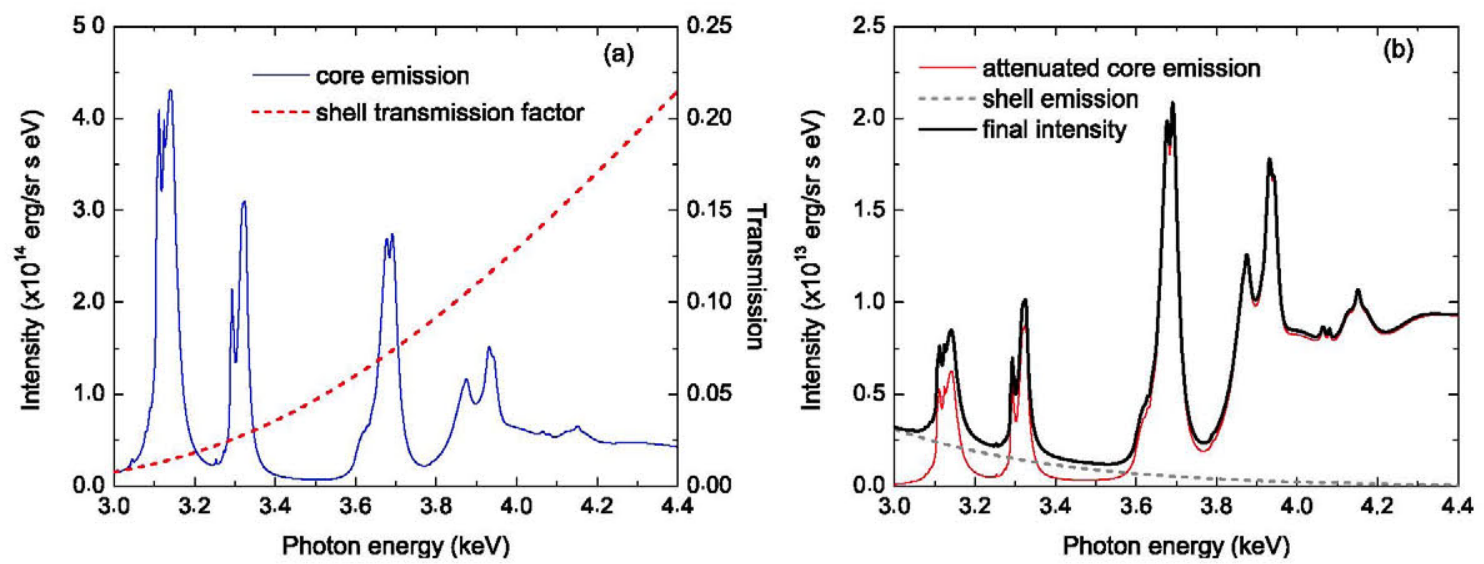

Figure 4. The sphere-slab model is used to illustrate the formation of the spectrum. Shell and core parameters values are $T_{\mathrm{s}}=285 \mathrm{eV}$, $N_{\mathrm{s}}=3.8 \times 10^{25} \mathrm{~cm}^{-3}, \Delta R=11 \mu \mathrm{m}, T_{\mathrm{c}}=930 \mathrm{eV}, N_{\mathrm{c}}=1.0 \times 10^{24} \mathrm{~cm}^{-3}, R=40 \mu \mathrm{m}$. (a) Core emission before being attenuated as it goes through the shell and shell transmission factor. $(b)$ Attenuated core emission, shell emission, and final emergent intensity. Figure adapted from [20]. Copyright (2011) by American Physical Society.

density conditions, ABAKO takes into account all nonautoionizing and autoionizing states consistent with the continuum-lowering. Radiation transport effects on level population kinetics were considered via escape factors [26]. Detailed Stark-broadened line shapes including the iondynamics effect [27-29] were used for parent and satellite transitions of the argon spectrum. For completeness, natural and Doppler line broadening were included as well, but they are small compared with Stark broadening. Additionally, a plasma broadening effect on the bound-free emissivity and opacity was included according to the approximation discussed in [30].

The model geometry shown was integrated numerically and employed to perform the data analysis. However, to gain insight into the model characteristics and the formation of the emission spectrum, we used a simplified model geometry, which corresponds to the case of a spherical plasma source (core) seen through a plasma slab (shell)-see figure $3(b)$. The advantage of this simplified model geometry is that it has the same underlying physics of the model in figure $3(a)$ but the radiation transport equation can be integrated analytically, namely,

$$
F^{v}=F_{\mathrm{c}}^{v} \mathrm{e}^{-\kappa_{\mathrm{s}}^{v} \Delta R}+F_{\mathrm{s}}^{v},
$$

with

$$
\begin{gathered}
F_{\mathrm{c}}^{v}=\pi R^{2} \frac{\varepsilon_{\mathrm{c}}^{\nu}}{\kappa_{\mathrm{c}}^{\nu}}\left[1+\frac{\mathrm{e}^{-2 \kappa_{\mathrm{c}}^{v} R}}{\kappa_{\mathrm{c}}^{\nu} R}-\frac{\left(1-\mathrm{e}^{-2 \kappa_{\mathrm{c}}^{v} R}\right)}{2\left(\kappa_{\mathrm{c}}^{\nu} R\right)^{2}}\right], \\
F_{\mathrm{s}}^{\nu}=\pi R^{2} \frac{\varepsilon_{\mathrm{s}}^{\nu}}{\kappa_{\mathrm{s}}^{\nu}}\left(1-\mathrm{e}^{-\kappa_{\mathrm{s}}^{\nu} \Delta R}\right),
\end{gathered}
$$

where $v$ is the photon frequency, $F^{v}$ is the final intensity flux and $\varepsilon_{\mathrm{c}}^{v}, \kappa_{\mathrm{c}}^{v}, \varepsilon_{\mathrm{s}}^{v}$ and $\kappa_{\mathrm{c}}^{v}$ stand for the temperature, density, and photon energy dependent emissivity and opacity of the core and the shell, respectively.

The first term in equation (1), $F_{\mathrm{c}}^{v} \mathrm{e}^{-\kappa_{\mathrm{s}}^{v} \Delta R}$, represents the radiation emitted by and transported through the core further attenuated by the transmission through the shell. The various contributions to $F_{\mathrm{c}}^{v}$ within the square brackets emerge after considering the intensity which results from the integral of the radiation transport equation computed along a chord inside a spherical, uniform plasma source and further integrating the intensity flux along the given line of sight over the surface of the sphere-details can be found in [31]. The intensity flux $F_{\mathrm{c}}^{v}$ has the small and large optical depth limits given by $F_{\mathrm{c}}^{\nu} \rightarrow$ $\frac{4 \pi}{3} R_{\mathrm{c}}^{3} \varepsilon_{\mathrm{c}}^{\nu}$ and $F_{\mathrm{c}}^{\nu} \rightarrow \pi R^{2} \frac{\varepsilon_{\mathrm{c}}^{\nu}}{\kappa_{\mathrm{c}}^{\nu}}$ for $2 \kappa_{\mathrm{c}}^{\nu} R_{\mathrm{c}} \ll 1$ and $2 \kappa_{\mathrm{c}}^{\nu} R_{\mathrm{c}} \gg 1$, respectively. As stated in [31], the physical interpretation of both limits is clear: in the optically thin case the final result is the volume integral of the emissivity, and in the high optical depth limit the result corresponds to a surface radiator of effective area given by the cross-section of the sphere.

The second term in equation (1), $F_{\mathrm{s}}^{v}$, gives the selfemission of the shell. Hence, the emergent intensity includes both the argon and the plastic emissions. Because the shell selfemission decreases with $h v$ mainly as $\exp \left(-h v / T_{\mathrm{s}}\right)$, its effect is mostly noticeable in the low energy side of the observed photon energy range. Figure 4 graphically illustrates the details of the formation of the spectrum according to equation (1) for a given set of model parameters.

Fitting the observed spectrum by means of a weighted least-squares minimization procedure yields both core and shell temperature and density conditions. Furthermore, from $N_{\mathrm{c}}, R, N_{\mathrm{s}}$ and $R$ the areal density $\rho R$ of the imploded target can also be obtained. Details of this procedure and comparisons with hydro-simulations and experimental data can be found in [20]. From the analysis of the space- and timeintegrated spectrum recorded in the referred shock-ignition experiments, the core and compressed shell temperature and density conditions values were extracted: $T_{\mathrm{c}}=930 \pm 5 \% \mathrm{eV}$, $N_{\mathrm{c}}=1.0 \times 10^{24} \pm 20 \% \mathrm{~cm}^{-3}, T_{\mathrm{s}}=285 \pm 6 \%$ and $N_{\mathrm{s}}=$ $3.8 \times 10^{25} \pm 25 \% \mathrm{~cm}^{-3}$. Uncertainties in the parameters were determined from a confidence interval statistical study that takes into account the correlations between model parameters [32]. Furthermore, the spectroscopic analysis also provides an estimate of $\rho R=0.17 \pm 20 \% \mathrm{~g} \mathrm{~cm}^{-2}$ for the target's areal density at the collapse of the implosion, which is consistent consistent with the results of early experiments based on particle diagnostics [22].

\section{Conclusions}

Fundamental research and modelling in plasma atomic physics play an important role to understand different phenomena in a 
wide variety of laboratory and astrophysical plasmas. In this regard, the codes and models developed by the Atomic Physics Group at the Institute of Nuclear Fusion (DENIM) combine accuracy and celerity in a proper balance and can be a valid tool to design and/or interpret experiments. As illustration, we discuss the use of the ABAKO/RAPCAL computational package, first, for the characterization of radiative blast waves launched in xenon clusters and, second, as part of the physical model for the spectroscopic diagnosis of shockignition implosions.

\section{Acknowledgments}

This work has been supported by the Spanish Ministry of Science and Innovation Grant No ENE2009-11208 and the Keep-in-Touch Project of the EU. The shock-ignition spectroscopic analysis was supported by Grant DE-FG5209NA29042 of the NLUF program of the US Department of Energy.

\section{References}

[1] Scott H A and Hansen S B 2010 Advances in NLTE modeling for integrated simulations High Energy Density Phys. $639-47$

[2] Bates R D, Kingston A E and McWirther R P W 1962 Recombination between electrons and atomic ions: I. Optically thin plasmas Proc. R. Soc. Lond. A 267 297-312

[3] Florido R, Rodríguez R, Gil J M, Rubiano J G, Martel P, Mínguez E and Mancini R C 2009 Modeling of population kinetics of plasmas that are not in local thermodynamic equilibrium, using a versatile collisional-radiative model based on analytical rates Phys. Rev. E 80056402

[4] Rodriguez R, Florido R, Gil J M, Rubiano J G, Martel P and Mínguez E 2008 RAPCAL code: a flexible package to compute radiative properties for optically thin and thick low and high-z plasmas in a wide range of density and temperature Laser Part. Beams 26 433-48

[5] Mendoza M A, Rubiano J G, Gil J M, Rodiguez R, Florido R, Martel P and Mínguez E 2011 A new set of relativistic screening constants for the screened hydrogenic model High Energy Density Phys. 7 169-79

[6] Ralchenko Yu, Kramida A E and Reader J NIST Atomic Spectra Database Website: physics.nist.gov/ PhysRefData/ASD

[7] Gu M F 2008 The flexible atomic code Can. J. Phys. $86675-89$

[8] Mendoza M A 2011 PhD Thesis Universidad de Las Palmas de Gran Canaria

[9] Magee N H et al 1995 ASP Conf. Ser. 7851

[10] Drake R P 2006 High-Energy-Density Physics: Fundamentals, Inertial Fusion and Experimental Astrophysics (Berlin: Springer)

[11] Remington B A, Drake R P and Ryutov D 2006 Experimental astrophysics with high power lasers and $\mathrm{Z}$ pinches Rev. Mod. Phys. 78 755-807

[12] Osterhoff J, Symes D R, Edens A D, Moore A S, Hellewell E and Ditmire T 2009 Radiative shell thinning in intense laser-driven blast waves New. J. Phys. 11023022

[13] Symes D R et al 2010 Investigations of laser-driven radiative blast waves in clustered gases High Energy Density Phys. $6274-9$

[14] Ditmire T, Shigemori K, Remington B A, Estabrook K and Smith R A 2000 The production of strong blast waves through intense laser irradiation of atomic clusters Astrophys. J. Suppl. Ser. 127 299-304

[15] Rodríguez R, Gil J M, Florido R, Rubiano J G, Mendoza M A, Martel P, Mínguez E, Symes D R, Hohenberger M and Smith R A 2011 Determination of the average ionization and thermodynamic regimes of xenon plasmas with an application to the characterization of blast waves launched in xenon clusters High Energy Density Phys. $771-6$

[16] Rodríguez R et al 2012 Determination and analysis of plasma parameters for simulations of radiative blast waves launched in clusters of xenon and krypton Plasma Phys. Control. Fusion 54045012

[17] Ryutov D, Drake R P, Kane J, Liang E, Remington B A and Wood-Vasey W M 1999 Similarity criteria for the laboratory simulation of supernova hydrodynamics Astrophys. J. 518 821-32

[18] Salzmann D 1998 Atomic Physics in Hot Plasmas (Oxford: Oxford University Press)

[19] Florido R, Mancini R C, Nagayama T, Tommasini R, Delettrez J A, Regan S P and Yaakobi B 2010 Spectroscopic modeling of an argon-doped shock-ignition implosion Rev. Sci. Intrum. 81 10E307

[20] Florido R, Mancini R C, Nagayama T, Tommasini R, Delettrez J A, Regan S P and Yaakobi B 2011

Measurements of core and compressed-shell temperature and density conditions in thick-wall target implosions at the omega laser facility Phys. Rev. E 83066408

[21] Betti R, Zhou C D, Anderson K S, Perkins L J, Theobald W and Solodov A A 2007 Shock ignition of thermonuclear fuel with high areal density Phys. Rev. Lett. 98155001

[22] Theobald W et al 2008 Initial experiments on the shock-ignition inertial confinement fusion concept Phys. Plasmas 15056306

[23] Perkins L J, Betti R, LaFortune K N and Williams W H 2009 Shock ignition: A new approach to high gain inertial confinement fusion on the national ignition facility Phys. Rev. Lett. 103045004

[24] Ribeyre X, Schurtz G, Lafon M, Galera S and Weber S 2009 Shock ignition: an alternative scheme for HiPER Plasma Phys. Control. Fusion 51015013

[25] Canaud B and Temporal M 2010 High-gain shock ignition of direct-drive ICF targets for the Laser Mégajoule New. J. Phys. 12043037

[26] Mancini R C, Joyce R F and Hooper C F Jr 1987 Escape factors for Stark-broadened line profiles J. Phys. B: At. Mol. Phys. 20 2975-87

[27] Haynes D A, Garber D T, Hooper C F, Mancini R C, Lee Y T, Bradley D K, Delettrez J A, Epstein R and Jaanimagi P A 1996 Effects of ion dynamics and opacity on Stark-broadened argon line profiles Phys. Rev. E 53 1042-50

[28] Woltz L A and Hooper C F 1988 Calculation of spectral-line profiles of multielectron emitters in plasmas Phys. Rev. A 38 4766-71

[29] Mancini R C, Kilcrease D P, Woltz L A and Hooper C F 1991 Calculational aspects of the Stark line broadening of multielectron ions in plasmas Comput. Phys. Commun. $63314-22$

[30] Iglesias E J and Griem H R 1996 Transparency window effect in the Inglis-Teller region of the BV Lyman series J. Quant. Spectrosc. Radiat. Transfer 55 383-9

[31] Burris-Mog T J et al 2006 Line broadening analysis of implosion core conditions at $\mathrm{z}$ using argon $\mathrm{k}$-shell spectroscopy J. Quant. Spectrosc. Radiat. Transfer 99 120-30

[32] Press W H, Teukolsky S A, Vetterling W T and Flannery B P 2007 Numerical Recipes: The Art of Scientific Computing 3rd edn (Cambridge: Cambridge University Press) 